\title{
Improving sensor network performance with wireless energy transfer
}

\section{Constantinos Marios Angelopoulos}

Centre Universitaire d'Informatique,

Université de Genève,

1227 Carouge, Geneva, Switzerland

Email: Marios.Angelopoulos@unige.ch

\section{Sotiris Nikoletseas, Theofanis P. Raptis* and Christoforos Raptopoulos}

Computer Technology Institute and Press 'Diophantus' (CTI) \&

Department of Computer Engineering and Informatics,

University of Patras,

26500 Patras, Greece

Email: nikole@cti.gr

Email: traptis@ceid.upatras.gr

Email: raptopox@ceid.upatras.gr

${ }^{*}$ Corresponding author

\section{Filippos Vasilakis}

School of Electrical Engineering, Royal Institute of Technology (KTH),

10044 Stockholm, Sweden

Email: fvas@kth.se

\begin{abstract}
Through recent technology advances in the field of wireless energy transmission wireless rechargeable sensor networks have emerged. In this new paradigm for wireless sensor networks a mobile entity called mobile charger (MC) traverses the network and replenishes the dissipated energy of sensors. In this work we first provide a formal definition of the charging dispatch decision problem and prove its computational hardness. We then investigate how to optimise the trade-offs of several critical aspects of the charging process. In the light of these optimisations, we study the impact of the charging process to the network lifetime for three characteristic underlying routing protocols. Finally, we propose a mobile charging protocol that locally adapts the circular trajectory of the MC to the energy dissipation rate of each sub-region of the network. We compare this protocol against several $\mathrm{MC}$ trajectories by a detailed experimental evaluation. The derived findings demonstrate significant performance gains.
\end{abstract}

Keywords: sensor networks; energy efficiency; wireless charging; wireless energy transfer; mobility; distributed algorithms; experimentation; performance.

Reference to this paper should be made as follows: Angelopoulos, C.M., Nikoletseas, S., Raptis, T.P., Raptopoulos, C. and Vasilakis, F. (2015) 'Improving sensor network performance with wireless energy transfer', Int. J. Ad Hoc and Ubiquitous Computing, Vol. 20, No. 3, pp.159-171.

Biographical notes: Constantinos Marios Angelopoulos is currently a Postdoctoral Scholar of excellence (boursier d'excellence) of the Swiss Confederation at the University of Geneva. Previously, he has worked as a Post-Doctoral Researcher at the Computer Technology Institute \& Press 'Diophantus'. He obtained his PhD from the Computer Engineering and Informatics Department of University of Patras, Greece under the supervision of Associate Professor S. Nikoletseas. His research interests include algorithmic aspects of wireless sensor networks and ad-hoc mobile computing, fundamental aspects of modern networking (focus on efficiency and reliability), distributed computing, algorithmic engineering and large scale simulation. He has co-authored several publications in international journals and refereed conferences and one invited chapter in a book published by Springer. 
Sotiris Nikoletseas is a Professor at the Computer Engineering and Informatics Department of Patras University, Greece and Director of the SensorsLab at CTI. His research interests include Algorithmic Techniques in Distributed Computing (focus on sensor and mobile networks), Probabilistic Techniques and Random Graphs, and Algorithmic Engineering. He has co-authored over 200 publications in journals and refereed conferences, several book chapters and two books (one on the probabilistic method and another on sensor networks), while he has delivered several invited talks and tutorials. He has served as the Program Committee Chair of many Conferences, and as Editorial Board Member of major journals. He has co-initiated international conferences on sensor networking. He has coordinated several externally funded European Union R\&D Projects related to fundamental aspects of modern networks.

Theofanis P. Raptis is a PhD student at the Department of Computer Engineering and Informatics, University of Patras, Greece. His research interests include algorithmic aspects of distributed computing and fundamental aspects of emerging networking concepts. His current research focuses on efficient and adaptive energy management in wireless sensor networks. He has co-authored several publications in acclaimed international refereed conferences and has participated in several European Union funded R\&D projects.

Christoforos Raptopoulos got his PhD entitled "Design and Analysis of Algorithms for Random and Evolutionary Networks" under the supervision on Professor Paul G. Spirakis, from the Computer Engineering and Informatics Department in the University of Patras, Greece, in 2008 (Evaluation Excellent). Between September 2008 and September 2009, he worked as a Postdoctoral at the University of Paderborn and Heinz Nixdorf Institute (HNI) in Germany. There he held the reading class "Markov Chains and Random Walks on Graphs", which several PhD students attended. In the academic year 2011-2012 he also held the course 'Algorithms and Complexity' for the Master's Program OSYL at the University of Patras. Currently, he is a Postdoctoral at the Research Academic Computer Technology Institute (RACTI). He is also a scholarship fellow ('Bourse d' excellence') at the Computer Science Department of the University of Geneva.

Filippos Vasilakis received his Diploma from the Department of Computer Engineering and Informatics of University of Patras, Greece, in 2012. He is currently pursuing his MSc in Networks Services and Systems in KTH, Sweeden. His research interests are in the areas of distributed systems and networks with a special interest in sensor networks.

This paper is a revised and expanded version of a paper entitled 'Efficient energy management in wireless rechargeable sensor networks' presented at the 15th ACM International Conference on Modeling, Analysis and Simulation of Wireless and Mobile Systems (MSWiM), Paphos, Cyprus, October 2012.

\section{Introduction and contribution}

Recent advances in the fields of wireless energy transfer and batteries material offer new possibilities for managing the available energy in WSNs. In the first field, the technology of highly-efficient wireless energy transfer was proposed for efficient, non-radiative energy transmission over midrange. The work in Kurs et al. (2007) and Karalis et al. (2008) has shown that through strongly coupled magnetic resonances, the efficiency of transferring 60 watts of power over a distance in excess of $2 \mathrm{~m}$ is as high as $40 \%$. Industry research also demonstrated that it is possible to improve transferring 60 watts of power over a distance of up to 1 meter with efficiency of 75\% (http://software.intel.com/enus/videos/ wireless-resonant-energy-link-wrel-demo). At present, commercial products utilising wireless energy transmission have been available on the market such as those in Powercast (http://www.powercastco.com/), in Murata Manufacturing (http://www.murata.com/) and in Texas Instruments (http://www.ti.com/).

Together with the research efforts on wireless energy transfer, several international organisations, such as the
Wireless Power Consortium (WPC, http://www.wireless powerconsortium.com/), the Alliance for Wireless Power (A4WP, http://www.a4wp.org/) and the Power Matters Alliance (PMA, http://www.powermatters.org/) aim at maximising the use of these technologies. WPC, an openmembership cooperation of Asian, European, and American companies in diverse industries, including electronics manufacturers and original equipment manufacturers, is working towards the global standardisation of wireless charging technology. A4WP, an independently operated organisation composed of global wireless power and technology industry leaders, focuses on a new wireless power transfer technology that provides spatial freedom for charging of electrical devices in cars, on table tops and for multiple devices simultaneously. PMA is a rapidly growing network of industry leaders working together to create a better power paradigm for battery equipped devices using wireless charging technology. The members come from a diverse set of industries including telecommunication, consumer devices, automotive, retail, furniture, surfaces and more.

In the batteries material field, there is the new battery material for ultra-fast charging. Ultra-fast charging was 
recently realised in $\mathrm{LiFePO}_{4}$ by creating a fast ion-conducting surface phase through controlled off-stoichiometry (Kang and Ceder, 2009). It inherits and combines the advantages of both conventional Li-ion batteries and super-capacitors, that bring high energy density. This way, the time to fully charge a battery is shortened to few seconds.

These new technologies lead the way towards a new paradigm for WSNs; the wireless rechargeable sensor networks (WRSNs). WRSNs consist of sensor nodes that may be either stationary or mobile, as well as few mobile nodes with high energy supplies. The latter, by using wireless energy transfer technologies are capable of fast charging sensor nodes. This way, the highly constrained resource of energy can be managed in great detail and more efficiently. Another important aspect is the fact that energy management in WRSNs can be performed passively from the perspective of sensor nodes and without the computational and communicational overhead introduced by complex energy management algorithms. Finally, WRSNs allow energy management to be studied and designed independently of the underlying routing protocol used for data propagation.

The problem. Let a WRSN consisting of a set of stationary sensor nodes and a special mobile node called mobile charger (MC). The sensor nodes are deployed uniformly at random over a network area and propagate data to a Sink using a routing protocol. The MC has finite energy supplies, that are significantly greater than those of a single sensor node, and is capable of charging the sensors. The problem we study is identifying the best possible configuration of the Mobile Charger in order to improve energy efficiency and to extend the lifetime of the network. Such MC configuration alternatives include the charging policy followed (i.e., whether to charge nodes fully or partially), the ratio of energy available to the charger over the energy initially deployed at the sensor nodes, as well as the optimisation of the network traversal by the charger e.g., finding which are the best trajectories the charger should follow. Although the problem might have some similarities with close research topics, such as data mules in sensor networks or sensor actor networks, the emerging tradeoffs necessitate the optimisation of the new aforementioned crucial parameters.

Our contribution. While considerable research efforts have been invested into energy-efficient scheduling of the MC, proposed solutions in the literature so far require a global (or at best, a semi-global) knowledge of the state of the network. On the contrary, the solutions proposed in this paper are fully distributed and adaptive, and rely solely on local information. Furthermore, our proposed protocol for the MC can be used in combination with any underlying routing protocol and adapts to the distribution of sensors in the network area.

More specifically, we investigate alternative strategies for efficient charging in WRSNs via a MC. First, we identify several critical aspects of the charging procedure and the corresponding trade-offs. We provide a formal definition of the charging problem and prove that it is computationally hard. We then try to optimise each trade-off and, lastly, we investigate the impact of the charging process over three characteristic families of routing protocols. The trade-offs we have identified include:

- $\quad$ how the total available energy of the network should be split between sensor nodes and the MC

- $\quad$ given that the energy the MC may deliver to the nodes is finite, whether each sensor will be fully or partially charged (and to what extent)

- what is the trajectory the MC should follow in order to charge the sensor nodes.

The implementation of the alternative strategies and their detailed simulation evaluation demonstrates significant performance gains with respect to several metrics such as network lifetime, connectivity and coverage, as well as energy balance properties.

\section{Related work and comparison}

In Zhao et al. (2011) the authors consider a sensor network in which a mobile entity is employed which (in contrast to our approach) serves also as a data collector and as an energy transporter that charges the static sensors on its migration tour. They provide a two-step approach: in the first step the mobile entity selects the maximum number of anchor points such that the sensors located in these anchor points hold the least energy and meanwhile the tour length is no more than a threshold. In the second step they formulate a utility maximisation problem on a flow-level network model in order to determine how to gather data from sensors. However this algorithm requires global information, thus making it not very practical in even medium-sized sensor networks. In Li et al. (2011) the authors analyse again the possibility of practical and efficient joint routing and charging schemes. They propose a sensor network in which both a MC and a base station appear. Each sensor periodically sends data hop-by-hop to the Sink using the collection tree protocol (CTP). Also, measurements of other local properties such as energy level, consumption rate, etc. are piggybacked along with data and reported to the Sink. Then, the base station, according to sensors information, schedules future charging activities and commands the MC through long range radio to execute the schedules. Authors show that the network lifetime is prolonged by the MC which mostly moves in energy-minimum paths. However, each sensor has to send more data to the Sink and the charger has to know the location of each sensor a priori. Authors in Shi et al. (2011) consider the scenario of a mobile charging vehicle periodically travelling inside the sensor network and charging the battery of each sensor node wirelessly. The necessary and sufficient conditions are introduced and the problem is studied as an optimisation problem, with the objective of maximising the ratio of the wireless charging vehicle's vacation time over the cycle time. However, in their model authors use global knowledge.

Overall, in all above methodologies the knowledge of the model (network topology, node generation rates, etc.) is much stronger than ours allowing for off-line and/or centralised 
optimisations under high levels of network information. Also, in several of these approaches the charging problem is coupled together with routing, while in our method the charging policy implicitly adapts to any underlying routing policy. This property is very important, since different routing alternatives may lead to different energy consumption patterns on the network. For example, an energy balance routing protocol leads to a more uniform overall energy consumption, whereas a hop-by-hop routing protocol tends to stress more the nodes that lie closer to the Sink.

Regarding the three families of routing protocols we use to investigate the impact of our methods, we refer to Heinzelman et al. (2000) for clustering, Chatzigiannakis et al. (2002) for routing and Jarry et al. (2006) and Efthymiou et al. (2006) for energy balanced data propagation.

Recently, there has been much research effort in WRSNs. In Zhao et al. (2011) the authors consider a sensor network where a mobile entity is employed which (in contrast to our approach) serves both as a data collector and as an energy transporter that charges the stationary sensors on its migration tour. They provide a two-step approach: in the first step the mobile entity selects the maximum number of anchor points such that the sensors located in these anchor points hold the least energy and meanwhile the tour length is no more than a threshold. In the second step they formulate a utility maximisation problem on a flow-level network model in order to determine how to gather data from sensors. This algorithm requires global information, thus making it not very practical in even medium-sized sensor networks.

In $\mathrm{Li}$ et al. (2011) the authors analyse again the possibility of practical and efficient joint routing and charging schemes. They propose a sensor network in which both a MC and a base station appear. Each sensor sends data hop-by-hop to the Sink periodically using the CTP (Gnawali et al., 2009). Also, measurements of other local properties such as energy level, consumption rate, etc. are piggybacked along with data and reported to the Sink. Then, the base station, according to sensors information, schedules future charging activities and commands the MC through long range radio to execute the schedules. Authors show that the network lifetime is prolonged by the MC which mostly moves in energy-minimum paths. However, each sensor has to send more data to the Sink and the charger has to know the location of each sensor a priori.

In Guo et al. (2013), the authors propose a framework of joint wireless energy replenishment and anchor-point based mobile data gathering in WSNs by considering various sources of energy consumption and time-varying nature of energy replenishment. They formulate a network utility maximisation problem which is constrained by flow conversation, energy balance, link and battery capacity and the bounded sojourn time of the mobile collector. and they present an algorithm composed of cross-layer data control, scheduling and routing components. In Shi et al. (2011) and Xie et al. (2012) the authors consider the scenario of a mobile charging vehicle periodically travelling inside the sensor network and wirelessly charging the battery of each sensor node. The necessary and sufficient conditions are introduced and the problem is studied as an optimisation problem, with the objective of maximising the ratio of the wireless charging vehicle's vacation time over the cycle time. Also, in Xie et al. (2013a, 2013b), the authors co-locate the mobile base station on the MC and minimise the energy consumption of the entire system while ensuring none of the sensor nodes runs out of energy. In contrast to our protocols, the models used in the above works use global knowledge.

In Peng et al. (2010) the authors build a proof-of-concept prototype by using a wireless power charger installed on a robot and sensor nodes equipped with wireless power receivers, carry out experiments on the prototype to evaluate its performance in small-scale networks of up to 10 nodes, and conduct simulations to study its performance in larger networks of up to a 100 nodes. Despite the fact that this paper nicely demonstrates the feasibility of a real, implemented WRSN, the simulations of the proposed heuristics are limited to a small number of sensor nodes in the network, an approach that is not convenient for highlighting the behaviour of the charging protocol in large scale networks.

In $\mathrm{Li}$ et al. (2012), the authors formulate an energyconstrained wireless charging problem, which maximises the number of sensors wirelessly charged by a MC. The paper proposes heuristic solutions based on the meta-heuristics of Particle Swarm Optimisation but, in contrast to our approach, the model assumes extensive knowledge on the charger and the performance evaluation is limited to simulations on smallscale networks.

Alternative versions of the problem have also attracted important research attention. In Zhang et al. (2012), Li et al. (2013), Madhja et al. (2013), Dai et al. (2013) and Wang et al. (2013) the authors consider the wireless charging problem, using multiple MCs. In this case, several other interesting aspects emerge, such as the minimum number of chargers that suffice to cover the network area, inter-charger coordination, etc. Another interesting approach is presented in $\mathrm{Fu}$ et al. (2013), where the charging process is conducted using another, RFID-based technology resulting in the introduction of the charging delay notion and different modelling of the problem.

Overall, in the majority of the above methodologies, the knowledge of the model is much stronger than ours, allowing for off-line and/or centralised optimisation under high levels of network information. Also, in several of these approaches the charging problem is coupled together with routing, while in our method the charging policy implicitly adapts to any underlying routing policy.

\section{The model}

\subsection{Deployment and energy model}

Our model features three types of devices: static sensors, a MC and one static Sink. We assume that there are $N$ sensors uniformly distributed at random in a circular area of radius $R$. The communication range of sensors (we denote it by $r$ ) varies according to requirements of the underlying routing protocol. The network density is

$$
\rho=\frac{N}{\pi R^{2}} .
$$


The Sink lies at the centre of the circular area. In our model we assume that the MC does not perform any data gathering process. For simplicity we assume that all sensors have the same data generation rate of $\lambda$ packets per unit time. This assumption is typical for scenaria were wireless sensor networks are used for periodical monitoring and reporting. Also we assume that $E_{\text {total }}$ is the total available energy in the network. Initially,

$$
E_{\text {total }}=E_{\text {sensors }}+E_{M C}^{\text {init }}
$$

where $E_{\text {sensors }}$ is the amount of energy shared among the sensor nodes and $E_{M C}^{\text {init }}$ is the total amount of energy that the MC may deliver to the network by charging sensors. At any given time the energy left to the $\mathrm{MC}$ for sensor charging is denoted as $E_{M C}^{\mathrm{curr}}$. The maximum amount of energy that a single sensor may store is denoted as $E_{\text {sensor }}^{\max }$ and is the initial energy given to each sensor, i.e.,

$$
E_{\text {sensor }}^{\max }=\frac{E_{\text {sensors }}}{N} \text {. }
$$

For transmitting and receiving a message, we assume that the radio module dissipates an amount of energy proportional to the message size. To transmit a k-bit message, the radio expends $E_{\tau}(k)=\epsilon_{\text {trans }} \cdot k$ and to receive a k-bit message, the radio expends $E_{R}(k)=\epsilon_{\mathrm{recv}} \cdot k$ where $\epsilon_{\text {trans }}$ and $\epsilon_{\text {recv }}$ are constants that depend on the radio module and the transmission range of the sensors. As usual, the power needed to transmit a message at distance $d$ is roughly $d^{\alpha}$ where $2 \leq \alpha \leq 6$ a constant; for simplicity we take $\alpha=2$.

\subsection{Charging model}

In our model the charging is performed point-to-point, i.e., only one sensor may be charged at a time from the MC by approaching it at a very close distance so that the charging process has maximum efficiency. The time that elapses while the MC moves from one sensor to another is considered to be very small when compared to the charging time; still the trajectory followed (and particularly its length) is of interest to us, since it may capture diverse cost aspects. We assume that the charging time is equal for every sensor and independent of its battery status.

\section{The charger dispatch decision problem}

Below we give a formal definition for the decision version of the problem we consider.

$C D D P$ : Suppose that we are given a set $S$ of sensors each one capable to store $E$ units of energy and for each sensor $s \in S$ a list $L_{s}$ of pairs $\left(t_{s}^{j}, e_{s}^{j}\right), j \geq 1$ in which $t_{s}^{j}$ corresponds to the time that the $j$ th message of $s$ was generated and $e_{s}^{j}$ the energy that the sensor used to transmit it. We are also given an $|S| \times|S|$ matrix $D$, where $D_{i, j}$ is the distance between sensors $i$ and $j$ and a MC $M$ which can charge a sensor in one time unit to its initial energy (notice that if we assume that the charger moves with constant speed $v$ then the time needed to travel between $i$ and $j$ is $\frac{D_{i, j}}{v}$ ). The charger dispatch decision problem (CDDP) is to determine whether there is a feasible schedule for $M$ to visit the sensors so that no message is lost due to insufficient energy.

Notice that we neglect to include energy needed by sensors in order to receive messages. Moreover, the messages a sensor $s$ might receive by other nodes are included in each $L_{s}$. Thus we suppose that these messages are generated by the sensor itself. This allows the consideration of different routing policies in a unified manner.

We show that the general version of the CDDP is $\mathcal{N} \mathcal{P}$ complete.

\section{Theorem 1: $C D D P$ is $\mathcal{N} \mathcal{P}$-complete.}

Proof: We first note that, given a certain walk $W$ of the charger visiting sensors in $S$, we can verify whether this walk is sufficient so that no message is lost, i.e., no message $x$ is generated on a sensor $s$ such that $x$ is the $j$ th message of $s$ and $s$ has less than $e_{s}^{j}$ available energy at time $t_{s}^{j}$. In particular, this can be done in $O(T \cdot|W|)$ time, where $T$ is the total number of events generated in the network. Therefore CDDP $\in \mathcal{N} \mathcal{P}$.

For the hardness part we use the Geometric Travelling Salesman Problem (G-TSP in short, see Garey and Johnson (1979), p.212). Let $P \subseteq \mathbb{Z} \times \mathbb{Z}$ and $B \in \mathbb{N}$ be the input of G-TSP. We now transform this into an input for CDDP as follows: We use a set $S$ of $|S|=|P|$ sensors and set $D_{i, j}$ equal to the Euclidean distance between the $i$ th and $j$ th point in $P$. Furthermore, for each sensor $s \in S$ we define its event list to be

$$
L_{s}=\left\{(0, E),\left(\frac{B}{v}, 1\right)\right\},
$$

where $v$ is the charger's speed. That is, two events happen in each sensor $s$, namely one at time 0 depleting all the energy available in $s$ and one at time $\frac{B}{v}$ requiring energy 1 . Notice that a solution to this instance of the CDDP problem would provide an answer to G-TSP, which means that G-TSP $\leq_{m}$ CDDP. This completes the proof.

\section{Trade-offs of the charging process}

Theorem 1 states that the problem is hard to solve, even with global knowledge of the energy dissipation. The heuristics that we present encounter an even harder problem, since the knowledge is limited to a local level. In order to properly understand and investigate the charging process we first analyse several of its aspects and specify their inherent tradeoffs.

\subsection{Energy percentage available to charger}

In order to be fair in our evaluation, we assume that the total available energy to the network $E_{\text {total }}$ is finite and same in all cases. This way, we will be able to investigate whether the energy efficiency is increased (and to what extent) with and without the introduction of the MC and the charging process to the network. This particular trade-off consists in how much 
energy (in terms of the total energy available) should the MC be initially equipped with. On the other hand, more energy to the MC leads to better online management of energy in the network. However, since $E_{\text {total }}=E_{\text {sensors }}+E_{M C}^{\text {init }}$, more energy to the $\mathrm{MC}$ also means that the sensor nodes will initially be only partially charged. Therefore, it will be more likely that they run out of energy before the MC charges them leading to possible network disconnection and low coverage of the network area. In order to determine the optimal energy amount available to charger as a percentage of $E_{\text {total }}$, we conduct experiments for various ratios between $E_{\text {sensors }}$ and $E_{M C}^{\text {init }}$.

A key conclusion is that a rather modest percentage of energy at the MC is a wise strategy (see Section 7.2 and Figure 3 ), with a $20 \%$ percentage value being the best choice.

\section{$5.2 \quad$ Full vs. partial charging}

Each time the MC visits a node a straightforward strategy would be to fully charge that node. This way the MC would maximise the time interval of revisiting the node before it runs out of energy. However, as the network operates, energy is dissipated in nodes due to data propagation and in the $\mathrm{MC}$ due to the charging process. Therefore, the MC will have increasingly less energy to distribute to more and more nodes.

Another approach is to judiciously spread the precious available energy to as many nodes as possible in order to extend the network lifetime. Following this rationale, the amount of energy the MC delivers to a node $i$ is proportional to the residual charging energy of the MC. More formally, MC charges a node until its energy becomes

$$
e_{i} \approx \frac{E_{M C}^{\mathrm{curr}}}{E_{M C}^{\mathrm{init}}} \cdot E_{\mathrm{sensor}}^{\mathrm{max}}
$$

In order to determine the best strategy we conduct detailed experiments comparing the full charging strategy against our adaptive, partial charging strategy.

Our basic result is that partial charging is more efficient than the full case (see Section 7.1 and Figure 2).

\section{Traversal strategies of the MC}

\subsection{Global knowledge traversal strategy}

The global-knowledge charger we study is an online solution that in each round minimises the product of each sensor's energy with its distance from the current position of the charger. More specifically, in each moving step the global charger minimises the following product:

$$
\min \left\{\left(1+\frac{E_{\text {curr }}}{E_{\text {init }}}\right) \cdot\left(1+\frac{\text { dist }_{\text {curr }}}{2 R}\right)\right\}
$$

where $E_{\text {curr }}, E_{\text {init }}$ and dist curr $_{\text {are respectively the current }}$ energy, initial energy and distance of each sensor, with the minimum taken over all sensors in the network (or at least a large part of it). Since this strategy requires a global knowledge of the state of the network, it is expected to outperform all other strategies, thus somehow representing a performance bound.
However, it would not be suitable for real life networks as it introduces great communication overhead (i.e., every node has to propagate its residual energy to the MC) and does not scale well with network size.

\subsection{Spiral traversal strategy}

Starting from the Sink, the MC traverses a path which forms a set of concentric circles, centred around the Sink but with increasing radii. Thus it forms a spiral until it reaches the boundaries of the network area. Then the MC follows the same path towards the opposite direction. The advantages of this movement is that due to its space filling attributes, the MC covers the whole network and almost every node is charged, until the energy of MC is totally depleted. On the other hand, this movement is not adaptive, i.e., it does not take into account differences to the energy depletion rates of each subregion of the network caused by the underlying routing protocols, such as bottleneck areas.

\subsection{Diameter traversal strategy}

Starting from the Sink, located at the centre of the circular network area, the MC arbitrarily chooses a direction and moves towards the perimeter of the network moving along the corresponding diameter. Once it has reached the edge of the network area, the MC traverses the perimeter of the network area for an angle of $d$ degrees, i.e., it moves over

$$
\frac{d}{2 \pi} 2 \pi R=d R
$$

length along the perimeter. Then, it starts moving again along the new corresponding diameter until it reaches the opposing edge of the network area. This procedure is repeated until there is no energy left in MC for charging. In order for the areas that the MC charges not to overlap, in every step $d$ is chosen uniformly at random from the interval of $(0,90]$ degrees. By following this trajectory pattern the MC manages to charge sensors that lie both close to and far from the Sink. However, although simple, it is also a non-adaptive strategy as well. An instance of the diameter traversal strategy is depicted in Figure 1.

Figure 1 Diameter traversal strategy

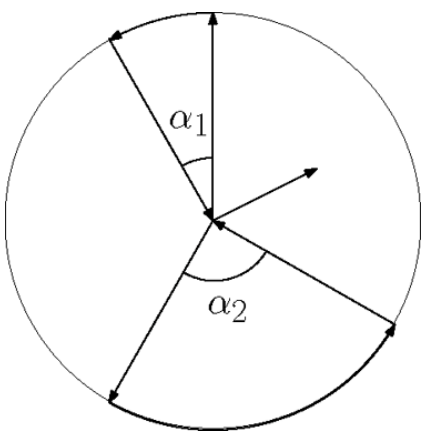

\subsection{Random walk traversal strategy}

In a simple, blind random walk, each next move of the MC is random and stochastically independent of the previous ones. 
Furthermore, given that the current node is $i$, the probability of moving to any neighbouring node $j$ is

$$
p_{i, j}=\frac{1}{\operatorname{deg}(i)} .
$$

This method is very robust, since it probabilistically guarantees that eventually all network regions and nodes will be visited, but it may become inefficient on networks with bottleneck areas, or under routing protocols with special nodes (like clustering protocols) or nodes that serve a lot of traffic, as in the case of multi-hop data propagation.

\subsection{Our adaptive circular traversal protocol}

Given the symmetric geometry, uniform density and uniform data generation rate of the network, we propose that the MC follows a circular trajectory around the Sink. The radius of the trajectory varies and adapts to the energy depletion rates of each subregion of the network. Starting from the Sink, the mobile charger traverses a path which forms a set of concentric circles, centred around the Sink with varying (increasing or decreasing) radii. In particular, the MC charges the sensors inside the ring which contains the corresponding trajectory. The width $d$ of each ring is pre-specified and constant. For example, if we denote by $S$ the set of the sensors to be charged at the $k$ th ring, then

$$
|S|=\frac{N}{R^{2}}\left((k d)^{2}-(k-1)^{2} d^{2}\right)=\frac{N}{R^{2}}\left(2 k d-d^{2}\right) .
$$

Let $e_{i}$ denote the current residual energy of node $i$. While at a given distance from the Sink, the MC records the mean value of the energy of the sensors lying on the corresponding circular trajectory; we denote this value by $\bar{E}_{\text {current }}$. Accordingly, the MC keeps record of the mean value of the energy of the sensors lying on its previous circular trajectory; we denote this value by $\bar{E}_{\text {previous }}$. Based on these two values, the MC tries to optimise its trajectory in terms of charging the nodes that deplete their energy faster. The protocol is shown below:

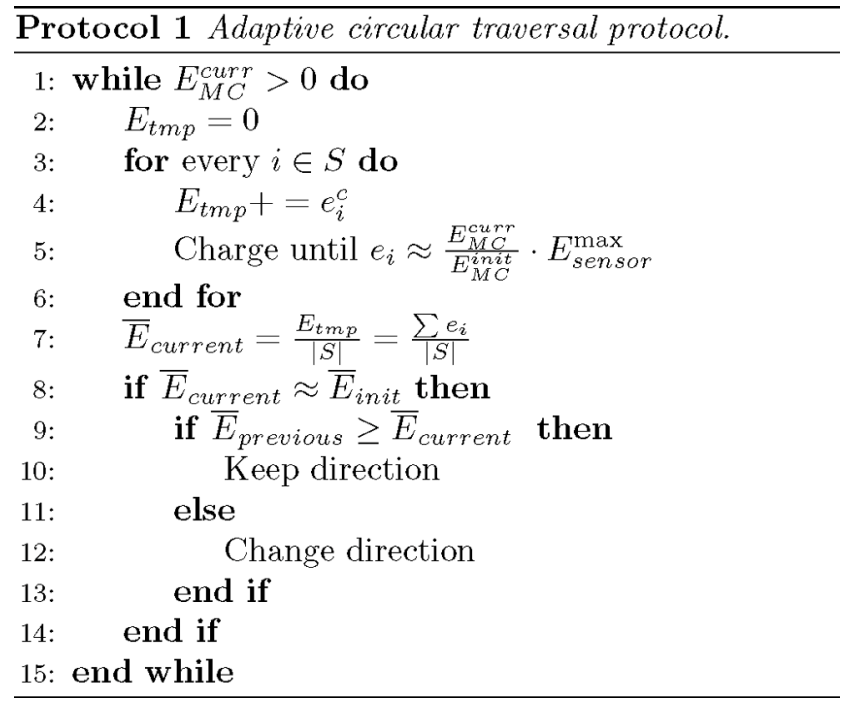

The MC commences traversing the network from the first centre of the network area by setting $R_{M C}=1$, i.e., it will first visit the nodes that lie one hop away from the Sink. Once all corresponding sensors are charged, i.e., the nodes the $\mathrm{MC}$ encounters have $e_{i} \simeq E_{\text {sensor }}^{\max }$, the $\mathrm{MC}$ increases its radius $R_{M C}$ by visiting nodes that are two hops away from the Sink. By comparing the values of $\bar{E}_{\text {current }}$ and $\bar{E}_{\text {previous }}$ the MC is able to figure out whether it moves towards areas that are stressed by the routing protocol or not. More specifically, if $\bar{E}_{\text {current }}<\bar{E}_{\text {previous }}$, then the MC assumes it is moving towards a stressed area of the network. Otherwise, if $\bar{E}_{\text {current }}>\bar{E}_{\text {previous, }}$, then it assumes it is moving away from the stressed areas and therefore should change direction.

\section{Experimental evaluation}

Our simulation environment for conducting the experiments is Matlab. The Sink is placed at the centre of the circular deployment area. For statistical smoothness, we apply the deployment of nodes in the network and repeat each experiment 100 times. For each experiment we simulate the data propagations that come from the generation of 5000 sensing events, and the average value is taken. The statistical analysis of the findings (the median, lower and upper quartiles, outliers of the samples) demonstrate very high concentration around the mean, so in the following figures we only depict average values.

Since the $N$ sensors are uniformly distributed in the circular area $\mathcal{A}$ of radius $R$, we apply a well known connectivity threshold, in order to maximise the probability that the produced random instances are connected. More strictly, since $\mathcal{A} \subset \mathbb{R}^{2}$, an instance of the random geometric graphs model $\mathcal{G}\left(\mathcal{X}_{N} ; r\right)$ is constructed as follows: select $N$ points $\mathcal{X}_{N}$ uniformly at random in $\mathcal{A}$. The set $V=\mathcal{X}_{N}$ is the set of vertices of the graph and we connect two vertices if their euclidean distance is at most $r$. In Gupta and Kumar (1998); Penrose (2003) it is shown that the connectivity threshold for $\mathcal{G}\left(\mathcal{X}_{N} ; r\right)$ is

$$
r_{c}=\sqrt{\frac{\ln N}{\pi N}} .
$$

In this paper we consider random instances of $\mathcal{G}\left(\mathcal{X}_{N} ; r\right)$ of varying density, by selecting

$$
r=\sqrt{\frac{c \ln N}{\pi N}},
$$

for different values of $c>1$, which guarantees that the produced random instance is connected with high probability. Throughout the experiments we fix the parameter $c$ to higher values for the Greedy routing protocol because it is more prone to early disconnections.

Since the network is dense enough, we assume that each transmission costs $r^{2}$ in terms of energy, where $r$ is the transmission range of a sensor node. Since the sensors are uniformly distributed and the event generation is also uniform, 
the average number of hops needed for an event to travel from the source node to the Sink is

$$
\frac{R}{2} \cdot \frac{1}{r}
$$

Consequently, the average energy spent for the routing of an event a source node to the Sink is

$$
r^{2} \frac{R}{2 r}=\frac{r R}{2}
$$

and for the overall energy spent in the network we have

$$
E_{\text {total }}=\frac{\mu r R}{2},
$$

where $\mu$ is the number of the events. We provide the network with

$$
E_{\text {total }}=\frac{h \mu r R}{4}
$$

for $h>1$, in order to prevent some nodes from dying during the experiments. The value of $E_{\text {total }}$ is the same across different simulation scenarios.

We focus on the following performance metrics:

- alive nodes over time, that is the number of nodes with enough residual energy to operate, during the progress of the experiment

- network energy map, which is a depiction of the whole network in terms of spatial aspects, after the generation of a number of events

- connectivity over time, in terms of the nodes' average degree during the progress of the experiment

- coverage ageing, that is the average coverage number (number of sensors having the point in their range) of 1000 randomly selected points in the network over time.

At first we investigate the above mentioned charging trade-offs and traversals by using the alive nodes over time as our lead metric. Then, in the light of these results, we apply the best possible configurations on the MC's parameters and conduct a detailed experimental analysis of the MC's impact of the charging process to each of the underlying routing protocols.

\subsection{Full vs. partial charging}

The first trade-off to be investigated is at which level should the sensor nodes be charged by the MC. We compare two possible strategies:

\section{- full charging of the nodes}

- partial charging of the nodes using our approach mentioned in Section 5.2.

At this stage we arbitrarily set the initial energy of the MC to be $40 \%$ of $E_{\text {total }}$. This ratio will be investigated individually and optimised later on. The results on the life evolution of the network are shown in Figure 2. Our partial charging strategy outperforms the full charging strategy, after a specific number of generated events, in all three routing protocols. This behaviour is the outcome of the fact that the MC is spending more energy in sensor charging when following the full charging strategy. Thus, the MC's energy is consumed earlier, resulting to a sharp increase in the node death rate.

\subsection{Energy available to the charger}

The next trade-off to be investigated is the optimal amount of total energy given to the charger with respect to the total energy of the network $E_{\text {total }}$. We conducted a comparison among several percentages of initial energy given to the charger. More specifically, we investigate the cases of 20, 40, 60, and $80 \%$ of the total energy to be given to the MC, using our partial charging strategy, since it is found to be more efficient. The results are shown in Figure 3.

It is clear that providing the MC with more than $40 \%$ of the total energy is negatively affecting the life evolution of the network, for all three routing protocols. This result comes from two facts. First, as $E_{\text {total }}=E_{\text {sensors }}+E_{M C}^{\text {init }}$, the more is the energy of the MC and the less is the initial energy of the nodes, resulting in a faster node death rate. A second corollary effect is that if the MC is provided with a high amount of energy, then it cannot distribute fast enough the whole amount of energy to the network, resulting in high residual energy at the MC by the time the network becomes disconnected.

\subsection{Traversals comparison}

Following the clarification of the previous questions, which resulted in an optimised configuration of our charger $(20 \%$ initial MC energy, partial charging strategy), comes an experimental comparison of the aforementioned possible traversal strategies of the MC.

More specifically, Figure 4 shows the impact on the life evolution of the network for each of the proposed strategies. The global knowledge charger outperforms, as expected, all the other chargers, until its energy is depleted. Given LEACH as the underlying routing protocol, the energy of the global knowledge charger is consumed earlier than our charger, resulting in a faster node death. The spiral charger, aside for the Greedy protocol, has a rather unstable impact on the number of alive nodes over time. This is explained by the distribution of the more stressed nodes over the network, for each routing protocol. More specifically, if we apply greedy routing, the nodes closer to the Sink tend to be more used, and the spiral charger's impact is positive, since these nodes are charged very frequently at the spiral traversal. On the contrary, for the $E_{i}$ and LEACH protocols, where more distant nodes are stressed, the charging frequency is reduced, due to the spiral traversal. Our charger's performance is very close to the powerful, global knowledge charger in all three cases and outperforms all other charging alternatives. In the LEACH case it actually becomes more efficient after a specific amount of events, since the global knowledge charger depletes its energy earlier.

The distance travelled by each charger during the experiments is given in Table 1. The random walk and diameter chargers are not only inefficient at managing the network 
Figure 2 Alive nodes over time for full and partial charging. The MC is provided with $40 \%$ of the total energy: (a) Greedy protocol; (b) $E_{i}$ protocol and (c) LEACH protocol

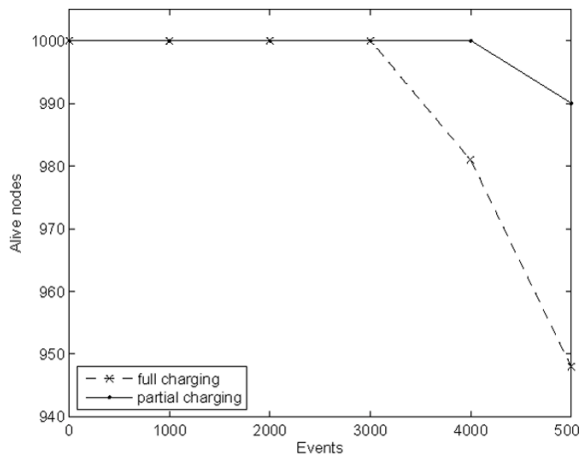

(a)

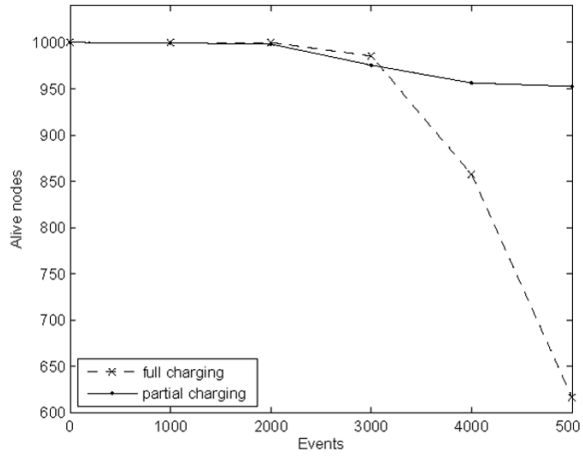

(b)

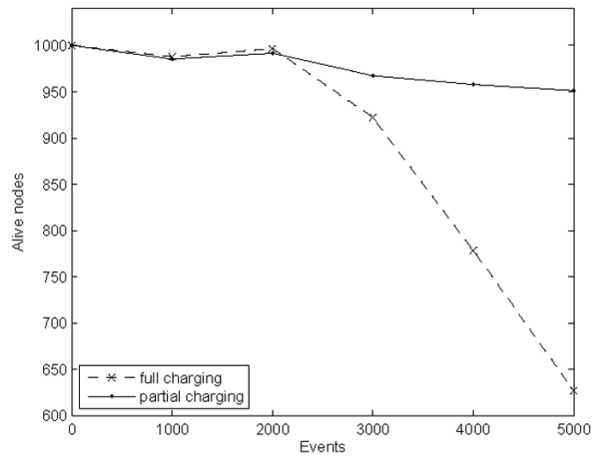

(c)

Figure 3 Alive nodes over time for various MC initial energy percentages. Partial charging is used: (a) Greedy protocol; (b) $E_{i}$ protocol and (c) LEACH protocol

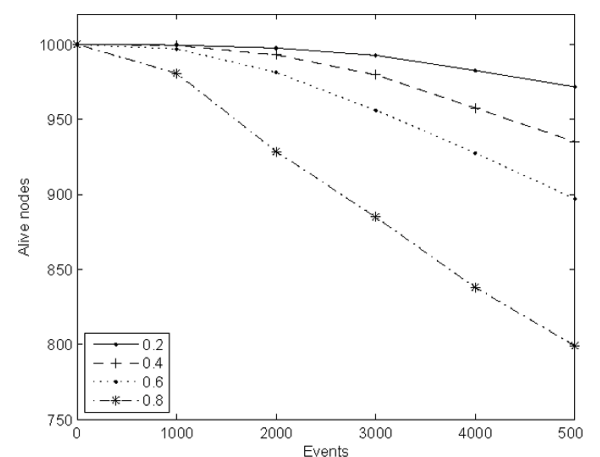

(a)

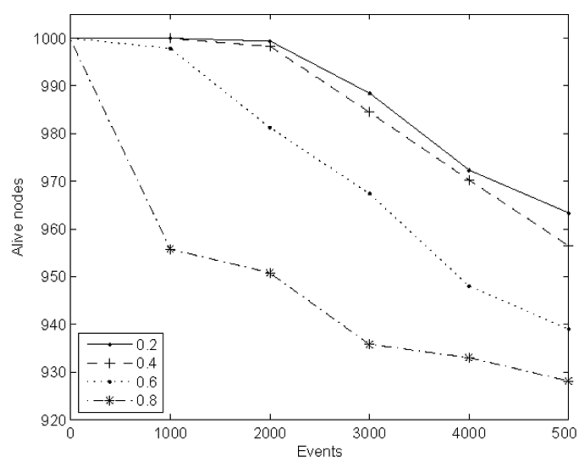

(b)

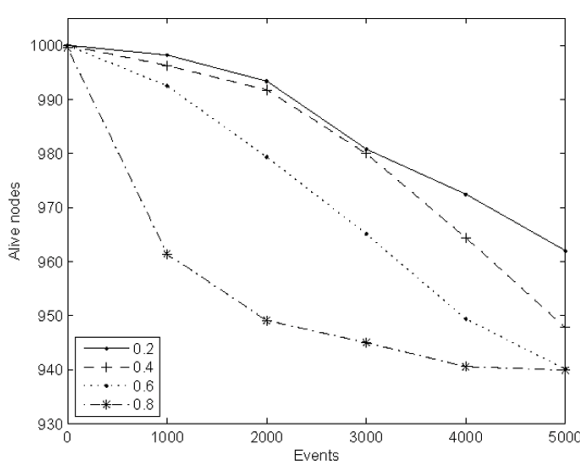

(c)

Figure 4 Alive nodes over time for various MC traversals, partial charging and 20\% initial energy: (a) Greedy protocol; (b) $E_{i}$ protocol and (c) LEACH protocol

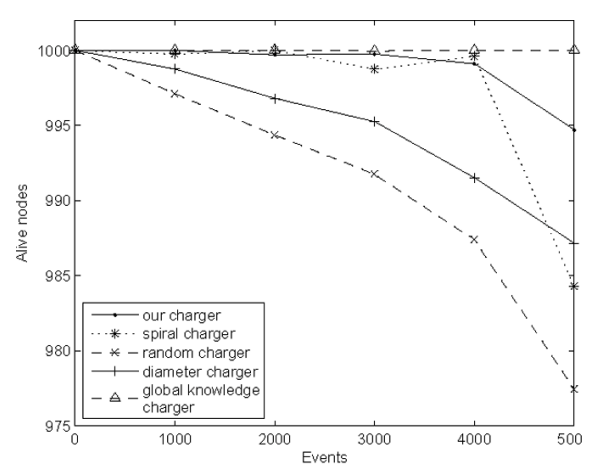

(a)

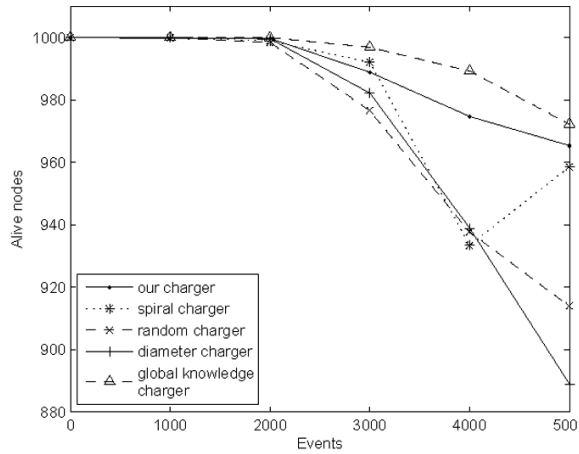

(b)

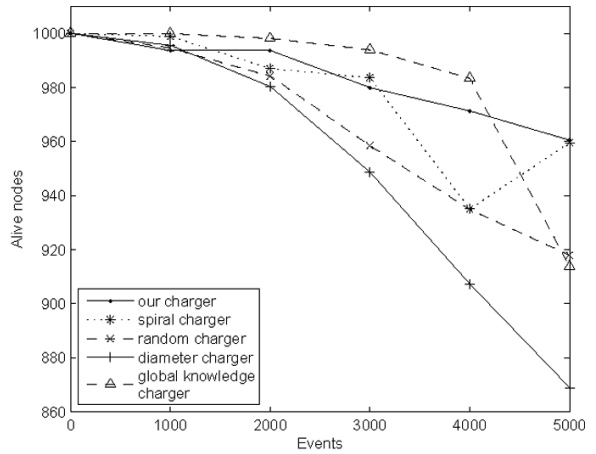

(c) energy but they also cover the greatest distance. Our charger travels significantly less distance than the spiral and the global knowledge charger.

\subsection{Overall improvements on the routing protocols}

After the line of experiments conducted, we are able to identify various parameters of the MC in order to achieve high performance. Following the previous results, the $\mathrm{MC}$ will be provided with $20 \%$ of the network's total energy, using our partial charging strategy and our adaptive traversal. We study the effect of our charger on the three routing protocols, on the number of alive nodes over time, the energy balance of the network, the average connectivity over time and the coverage ageing.

The overall death rate (in terms of alive nodes over time) of the network is vastly reduced in all three protocols, as shown in Figure 5.

Figures 6-8 depict the energy map of the network over time. More specifically, we present graphically the spatial evolution of energy dissipation in the network after 5000 event generations. Nodes with low residual energy are depicted with dark colours. In contrast, nodes with high residual energy are depicted with bright colours. The energy balance property is 
Figure 5 Alive nodes over time with and without charger: (a) Greedy protocol; (b) $E_{i}$ protocol and (c) LEACH protocol

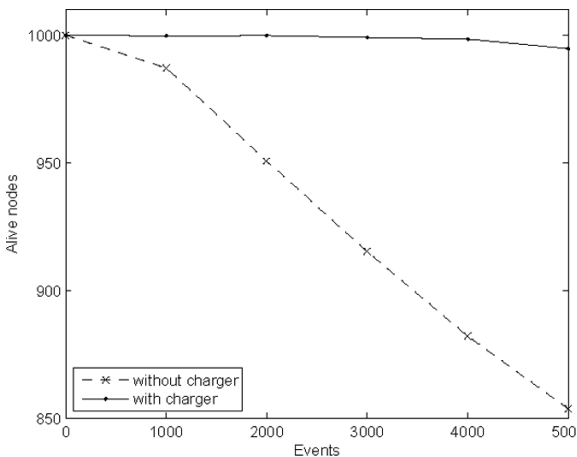

(a)

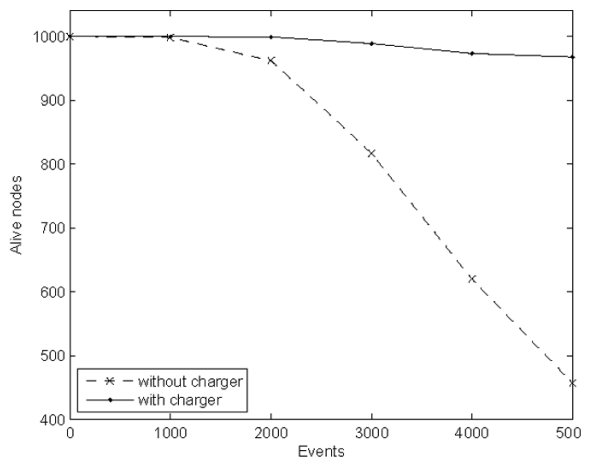

(b)

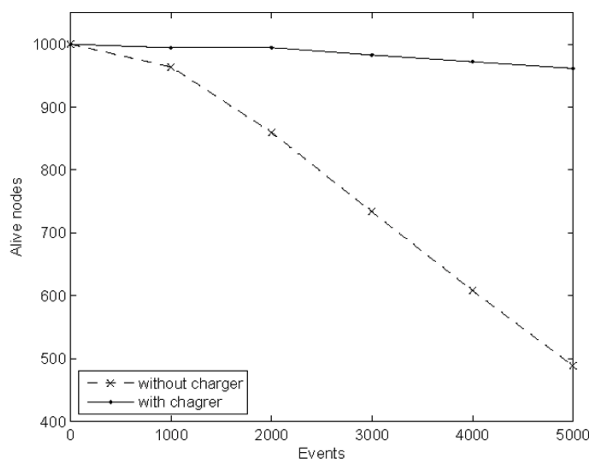

(c) crucial for the networks lifetime, since early disconnections are avoided and nodes tend to die in a uniform manner.

For all three protocols, we observe that the use of our charger improves the energy balance property. For the Greedy protocol, the stressed nodes closer to the Sink are used very frequently, thus their energy is depleted quickly. Using the charger, not only do the inner nodes not die that fast, but the network's energy in total is more balanced. For the $E_{i}$ and LEACH protocols, in which more distant nodes tend to be overused, the energy dissipation is higher at the distant nodes from the Sink. The use of charger has a similar effect with the Greedy case, balancing the network by charging more distant nodes from the Sink.

Table 1 Distance travelled by chargers (metres)

\begin{tabular}{lrrr}
\hline & Greedy & \multicolumn{1}{c}{$E_{i}$} & LEACH \\
\hline Global knowledge & 48.974 & 64.330 & 57.458 \\
Spiral & 64.167 & 64.167 & 64.167 \\
Random walk & 181.135 & 222.462 & 222.447 \\
Diameter & 152.252 & 172.734 & 173.169 \\
Our charger & 42.412 & 37.856 & 38.641 \\
\hline
\end{tabular}

Figure 6 Energy map for Greedy protocol: (a) without charger and (b) with charger

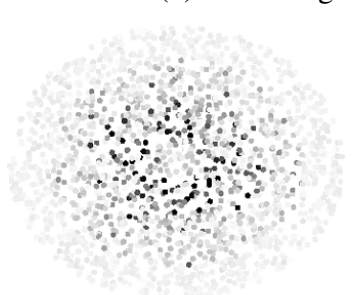

(a) (b)

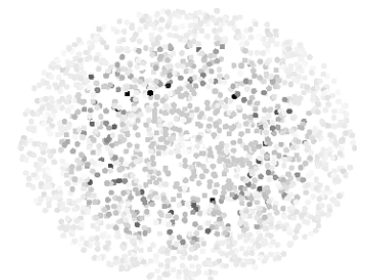

Connectivity is critical for sensor networks, as information collected needs to be sent to remote control centres. This is only possible if there is a path from each node to that centre. The connectivity of a sensor network is usually studied by considering a graph associated with that network. As far as connectivity is concerned, the average node degree over time is depicted in Figure 9. We observe that the degree number degradation follows a pattern identical to the node death rate in Figure 5.

Figure 7 Energy map for $E_{i}$ protocol: (a) without charger and (b) with charger

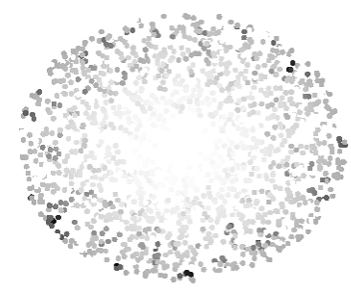

(a)

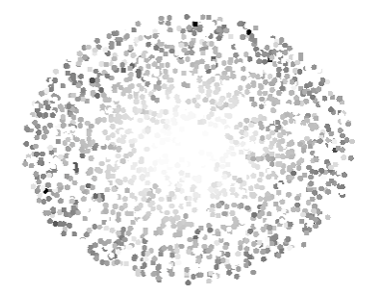

(b)
Figure 8 Energy map for LEACH protocol: (a) without charger and (b) with charger

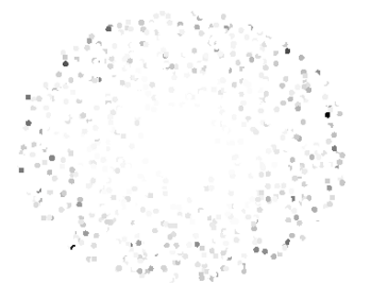

(a)

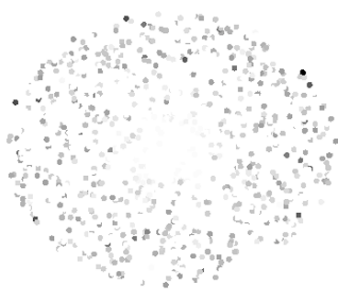

(b)
Point coverage problem is regarding how to ensure that each of some selected points in the network are covered by enough sensors. Coverage is an important aspect in sensor networks (e.g., localisation, data accuracy etc.). A point that is covered by $k$ sensors is called $k$-covered. The coverage ageing of 1000 randomly selected points in the network is shown in Figures 10-12. We examine how many points are $<2,3,>3$ covered for 5000 generated events. Each bar in the plots represents the number of the covered points. We observe that during the experiment without charger, the number of $<2$-covered points is increasing and the number of $>3$-covered points is decreasing. The use of the chargeris improving the coverage ageing of the selected points. More specifically, we observe that the absolute difference of the number of $<2$-covered points and $>3$-covered points, 
Figure 9 Average node degree over time with and without charger: (a) Greedy protocol; (b) $E_{i}$ protocol and (c) LEACH protocol

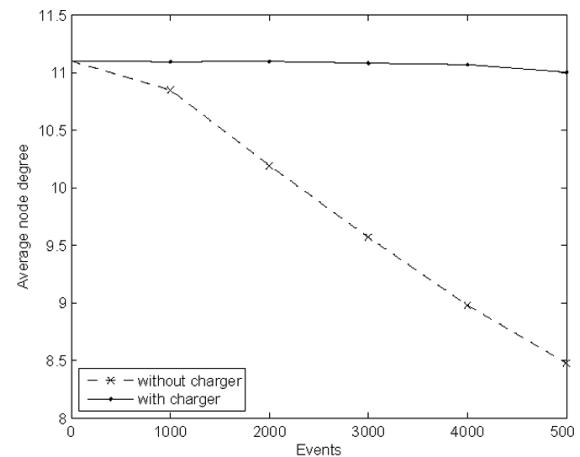

(a)

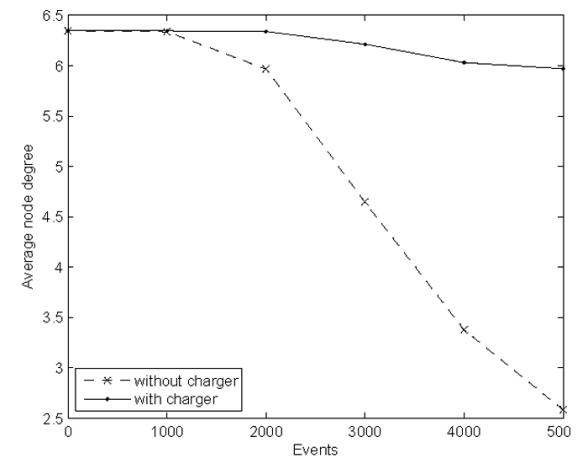

(b)

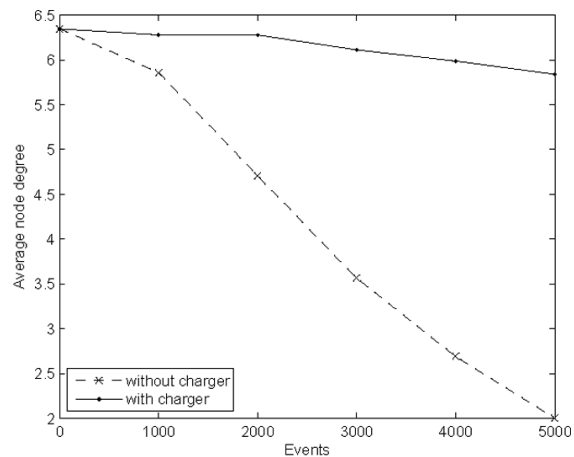

(c) between different time instances of the experiment, is not increasing quickly, compared to the experiment without the charger.

Figure 10 Coverage ageing for Greedy protocol: (a) without charger and (b) with charger

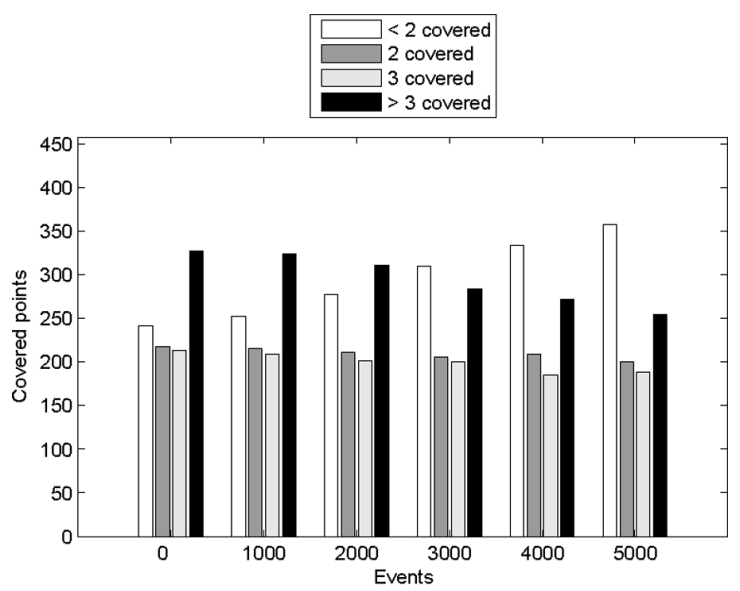

(a)

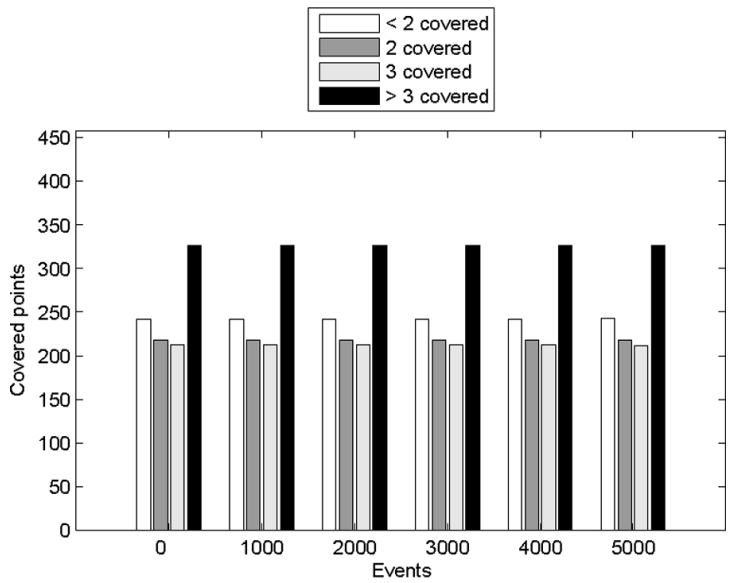

(b)
Figure 11 Coverage ageing for $E_{i}$ protocol: (a) without charger and (b) with charger

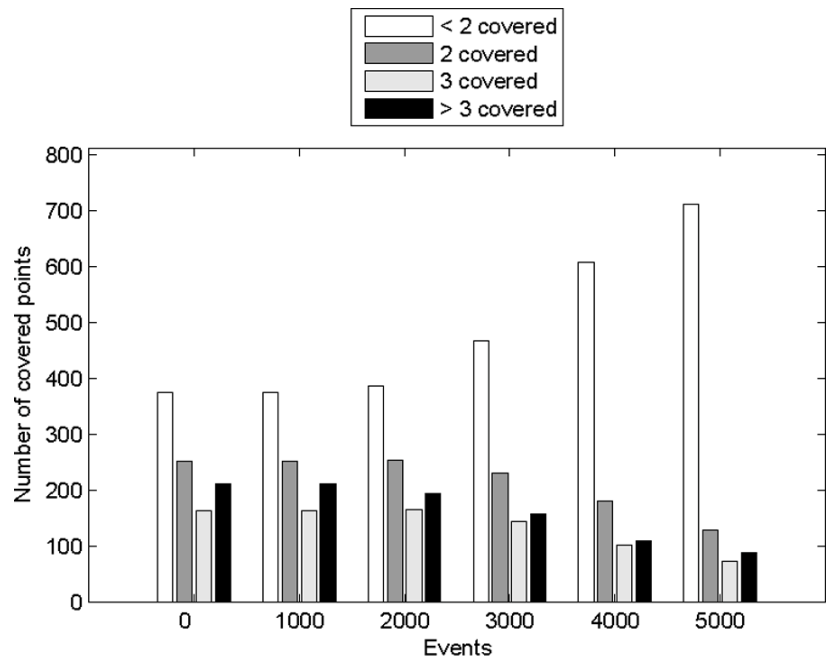

(a)

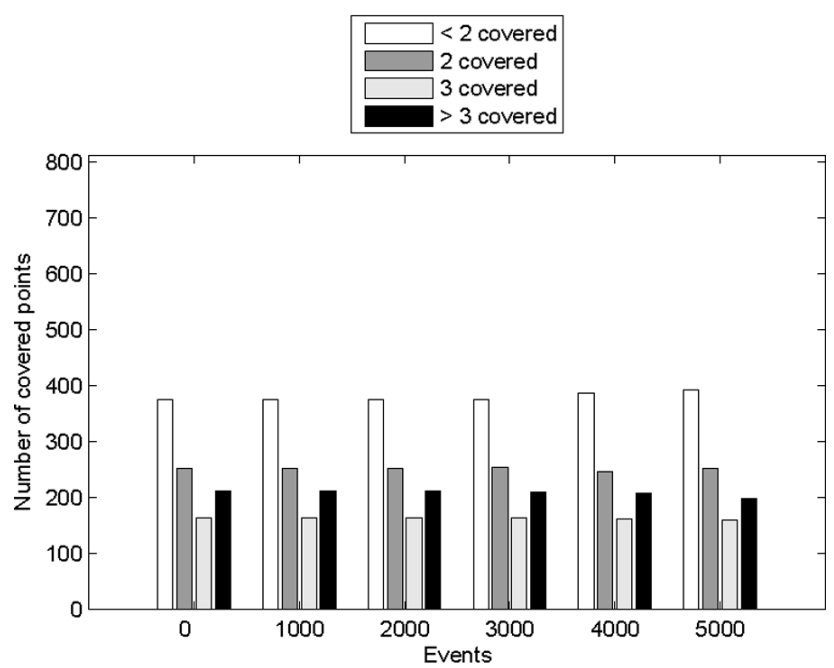

(b) 
Figure 12 Coverage ageing for LEACH protocol: (a) without charger and (b) with charger

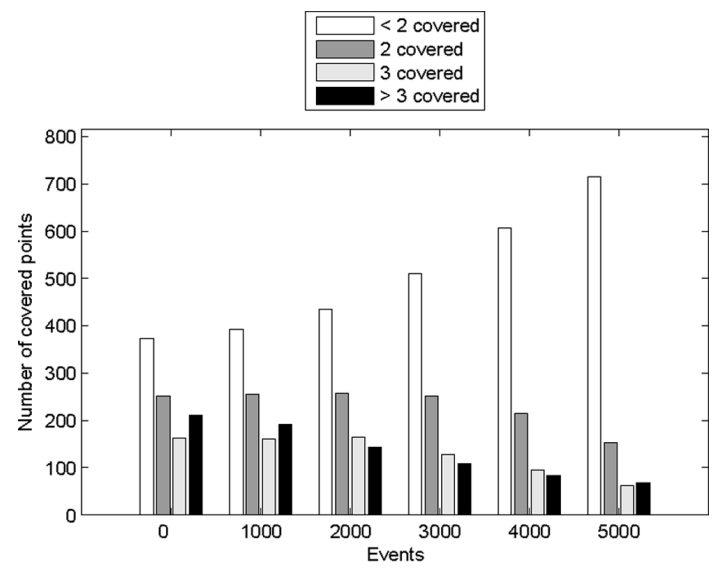

(a)

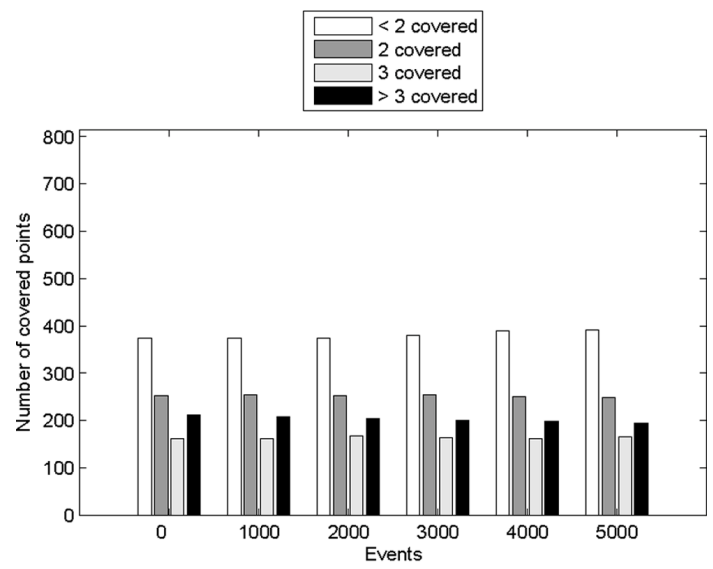

(b) partial charge of the nodes, by a charger with a moderate fraction of the total available energy, which follows a circular trajectory around the Sink of a radius which locally adapts to spatial variations of energy in the network. In particular, our simulation findings suggest significant performance gains with respect to various metrics such as network lifetime, connectivity and coverage as well as energy balance.

Future research directions include the design and rigorous analysis of efficient approximation algorithms with provable performance guarantees. In this respect, we note that the reduction used in Theorem 1 for proving the computational hardness of the problem is not preserving the approximation ratios (i.e., known efficient algorithms for the geometric TSP problem may not be very efficient for the charging problem), so this task seems quite challenging.

Another research direction is the modelling, analysis and simulation of other proposed charging models, such as the simultaneous charging of more than one sensor. Success ratio will also be considered as an additional metric, since the impact of the charger on the network's lifetime guarantees improvements on the aspect of lost messages.

\section{Acknowledgements}

This research was partially supported by

- the EU/FIRE IoT Lab project - STREP ICT-610477

- $\quad$ the European Social Fund (ESF) and Greek national funds through the Operational Program "Education and Lifelong Learning" of the National Strategic Reference Framework (NSRF) - Research Funding Program: Thalis-DISFER, Investing in knowledge society through the European Social Fund.

\section{References}

Chatzigiannakis, I., Nikoletseas, S. and Spirakis, P. (2002) 'Smart dust protocols for local detection and propagation', Proceedings of the Second ACM International Workshop on Principles of Mobile Computing', POMC '02, pp.9-16.

Dai, H., Wu, X., Xu, L., Chen, G. and Lin, S. (2013) 'Using minimum mobile chargers to keep large-scale wireless rechargeable sensor networks running forever', 22nd International Conference on Computer Communications and Networks (ICCCN), pp.1-7.

Efthymiou, C., Nikoletseas, S.E. and Rolim, J.D. (2006) 'Energy balanced data propagation in wireless sensor networks', Wireless Networks Vol. 12, No. 6, pp.691-707.

$\mathrm{Fu}$, L., Cheng, P., Gu, Y., Chen, J. and He, T. (2013) 'Minimizing charging delay in wireless rechargeable sensor networks', INFOCOM, Proceedings IEEE, pp.2922-2930.

Garey, M.R. and Johnson, D.S. (1979) Computers and Intractability: A Guide to the Theory of NP-Completeness, W.H. Freeman and Co., New York, NY, USA.

Gnawali, O., Fonseca, R., Jamieson, K., Moss, D. and Levis, P. (2009) 'Collection tree protocol', Proceedings of the 7th ACM Conference on Embedded Networked Sensor Systems, SenSys, pp.1-14.
For each of these issues we investigate (via detailed in each case. Overall, we show that the best strategy is 
Guo, S., Wang, C. and Yang, Y. (2013) 'Mobile data gathering with wireless energy replenishment in rechargeable sensor networks', INFOCOM, Proceedings IEEE, pp.1932-1940.

Gupta, P. and Kumar, P. (1998) 'Critical power for asymptotic connectivity', Proceedings of the 37th IEEE Conference on Decision and Control, 1998, Vol. 1, pp.1106-1110.

Heinzelman, W., Chandrakasan, A. and Balakrishnan, H. (2000) Energy-efficient communication protocol for wireless microsensor networks', Proceedings of the 33rd Annual Hawaii International Conference on System Sciences, 2000, Vol. 2, p.10.

Jarry, A., Leone, P., Powell, O. and Rolim, J. (2006) 'An optimal data propagation algorithm for maximizing the lifespan of sensor networks', Distributed Computing in Sensor Systems (DCOSS), Springer Berlin Heidelberg, Vol. 4026, pp.405-421.

Kang, B. and Ceder, G. (2009) 'Battery materials for ultrafast charging and discharging', Nature, Vol. 458, No. 7235, pp.190-193.

Karalis, A., Joannopoulos, J. and Soljacic, M. (2008) 'Efficient wireless non-radiative mid-range energy transfer', Annals of Physics, Vol. 323, No. 1, January Special Issue, pp.34-48.

Kurs, A., Karalis, A., Moffatt, R., Joannopoulos, J., Fisher, P. and Soljacic, M. (2007) 'Wireless power transfer via strongly coupled magnetic resonances', Science, Vol. 317, No. 5834, pp. $83-86$.

Li, J., Wang, C., Ye, F. and Yang, Y. (2013) 'Netwrap: an ndn based real time wireless recharging framework for wireless sensor networks', IEEE 10th International Conference on Mobile Adhoc and Sensor Systems (MASS), pp.173-181.

Li, K., Luan, H. and Shen, C-C. (2012) 'Qi-ferry: energy-constrained wireless charging in wireless sensor networks', Wireless Communications and Networking Conference (WCNC), IEEE, pp.2515-2520.

Li, Z., Peng, Y., Zhang, W. and Qiao, D. (2011) 'J-roc: a joint routing and charging scheme to prolong sensor network lifetime', 19th IEEE International Conference on Network Protocols (ICNP), pp.373-382.

Madhja, A., Nikoletseas, S. and Raptis, T.P. (2013) 'Efficient, distributed coordination of multiple mobile chargers in sensor networks', Proceedings of the 16th ACM International Conference on Modeling, Analysis and Simulation of Wireless and Mobile Systems, MSWiM, pp.101-108.

Peng, Y., Li, Z., Zhang, W. and Qiao, D. (2010) 'Prolonging sensor network lifetime through wireless charging', Real-Time Systems Symposium (RTSS), IEEE 31st, pp.129-139.
Penrose, M. (2003) Random Geometric Graphs, Oxford University Press, USA.

Shi, Y., Xie, L., Hou, Y. and Sherali, H. (2011) 'On renewable sensor networks with wireless energy transfer', INFOCOM, Proceedings IEEE, pp.1350-1358.

Wang, C., Li, J., Ye, F. and Yang, Y. (2013) 'Multi-vehicle coordination for wireless energy replenishment in sensor networks', IEEE 27th International Symposium on Parallel Distributed Processing (IPDPS), pp.1101-1111.

Xie, L., Shi, Y., Hou, Y., Lou, W., Sherali, H. and Midkiff, S. (2013a) 'Bundling mobile base station and wireless energy transfer: modeling and optimization', INFOCOM, Proceedings IEEE, pp.1636-1644.

Xie, L., Shi, Y., Hou, Y.T. and Sherali, H.D. (2012) 'Making sensor networks immortal: an energy-renewal approach with wireless power transfer', IEEE/ACM Trans. Netw., Vol. 20, No. 6, pp. $1748-1761$.

Xie, L., Shi, Y., Hou, Y.T., Lou, W. and Sherali, H.D. (2013b) 'On traveling path and related problems for a mobile station in a rechargeable sensor network', Proceedings of the Fourteenth ACM International Symposium on Mobile Ad Hoc Networking and Computing, MobiHoc, pp.109-118.

Zhang, S., Wu, J. and Lu, S. (2012) 'Collaborative mobile charging for sensor networks', IEEE 9th International Conference on Mobile Adhoc and Sensor Systems (MASS), pp.84-92.

Zhao, M., Li, J. and Yang, Y. (2011) 'Joint mobile energy replenishment and data gathering in wireless rechargeable sensor networks', 23rd International Teletraffic Congress (ITC), pp.238-245.

\section{Websites}

Alliance for Wireless Power, http://www.a4wp.org/

Powercast, http://www.powercastco.com/

Power Matters Alliance, http://www.powermatters.org/

Texas Instruments, http://www.ti.com/

The Wireless Power Consortium, http://www.wirelesspower consortium.com/

Murata Manufacturing, http://www.murata.com/ 